\title{
Both 3-T dGEMRIC and Acetabular-Femoral T2 Difference May Detect Cartilage Damage at the Chondrolabral Junction
}

\author{
Tobias Hesper MD, Evgeny Bulat MA, Sarah Bixby MD, \\ Alireza Akhondi-Asl PhD, Onur Afacan PhD, Patricia Miller MS, \\ Garrett Bowen BS, Simon Warfield PhD, Young-Jo Kim MD, PhD
}

Published online: 2 November 2016

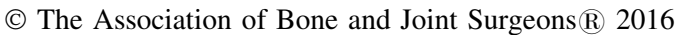

\begin{abstract}
Background In addition to case reports of gadoliniumrelated toxicities, there are increasing theoretical concerns about the use of gadolinium for MR imaging. As a result, there is increasing interest in noncontrast imaging techniques for biochemical cartilage assessment. Among them,
\end{abstract}

Each author certifies that he or she, or a member of his or her immediate family, has no funding or commercial associations (eg, consultancies, stock ownership, equity interest, patent/licensing arrangements, etc) that might pose a conflict of interest in connection with the submitted article.

All ICMJE Conflict of Interest Forms for authors and Clinical Orthopaedics and Related Research ${ }^{\circledR}$ editors and board members are on file with the publication and can be viewed on request.

Clinical Orthopaedics and Related Research ${ }^{\mathbb{R}}$ neither advocates nor endorses the use of any treatment, drug, or device. Readers are encouraged to always seek additional information, including FDAapproval status, of any drug or device prior to clinical use.

Each author certifies that his or her institution approved the human protocol for this investigation, that all investigations were conducted in conformity with ethical principles of research, and that informed consent for participation in the study was obtained.

T. Hesper, E. Bulat, P. Miller, G. Bowen, Y.-J. Kim ( $\square)$ Department of Orthopedic Surgery, Boston Children's Hospital, 300 Longwood Avenue, Boston, MA 02115, USA

e-mail: young-jo.kim@ childrens.harvard.edu

S. Bixby, O. Afacan, S. Warfield

Department of Radiology, Boston Children's Hospital, Boston, MA, USA

\section{A. Akhondi-Asl}

Division of Critical Care Medicine, Department of Anesthesiology, Perioperative and Pain Medicine, Boston

Children's Hospital, Boston, MA, USA

A. Akhondi-Asl

Harvard Medical School, Boston, MA, USA
T2 mapping holds promise because of its simplicity, but its biophysical interpretation has been controversial.

Questions/purposes We sought to determine whether (1) 3-T delayed gadolinium-enhanced MRI of cartilage (dGEMRIC) and T2 mapping are both capable of detecting cartilage damage at the chondrolabral junction in patients with femoroacetabular impingement (FAI); and (2) whether there is a correlation between these two techniques for acetabular and femoral head cartilage assessment.

Methods Thirty-one patients with hip-related symptoms resulting from FAI underwent a preoperative 3-T MRI of their hip that included dGEMRIC and T2 mapping (symptomatic group, 16 women, 15 men; mean age, $27 \pm 8$ years). Ten volunteers with no symptoms according to the WOMAC served as a control (asymptomatic group, seven women, three men; mean age, $28 \pm 3$ years). After morphologic cartilage assessment, acetabular and femoral head cartilages were graded according to the modified Outerbridge grading criteria. In the midsagittal plane, singleobserver analyses of precontrast $\mathrm{T} 1$ values (volunteers), the dGEMRIC index ( $\mathrm{T} 1_{\mathrm{Gd}}$, patients), and $\mathrm{T} 2$ mapping values (everyone) were compared in acetabular and corresponding femoral head cartilage at the chondrolabral junction of each hip by region-of-interest analysis.

Results In the symptomatic group, $\mathrm{T} 1_{\mathrm{Gd}}$ and $\mathrm{T} 2$ values were lower in the acetabular cartilage compared with corresponding femoral head cartilage $\left(\mathrm{T}_{\mathrm{Gd}}: 515 \pm 165 \mathrm{~ms}\right.$ versus $650 \pm 191 \mathrm{~ms}, \mathrm{p}<0.001 ; \mathrm{T} 2: 39 \pm 8 \mathrm{~ms}$ versus 46 $\pm 10 \mathrm{~ms}, \mathrm{p}<0.001)$. In contrast, the asymptomatic group demonstrated no differences in $\mathrm{T} 1$ and $\mathrm{T} 2$ values for the acetabular and femoral cartilages with the numbers available (T1: $861 \pm 130 \mathrm{~ms}$ versus $860 \pm 182 \mathrm{~ms}, \mathrm{p}=0.98$; $\mathrm{T} 2$ : $43 \pm 7 \mathrm{~ms}$ versus $42 \pm 6 \mathrm{~ms}, \mathrm{p}=0.73$ ). No correlation with the numbers available was noted between the modified 
Outerbridge grade and $\mathrm{T} 1, \mathrm{~T} 1_{\mathrm{Gd}}$, or $\mathrm{T} 2$ as well as between $\mathrm{T} 2$ and either $\mathrm{T} 1$ or $\mathrm{T} 1_{\mathrm{Gd}}$.

Conclusions Without the need for contrast media application, T2 mapping may be a viable alternative to dGEMRIC when assessing hip cartilage at the chondrolabral junction. However, acquisition-related phenomena as well as regional variations in the microstructure of hip cartilage necessitate an internal femoral head cartilage control when interpreting these results.

Level of Evidence Level IV, diagnostic study.

\section{Introduction}

In femoroacetabular impingement (FAI), structural abnormalities of the proximal femur (cam-type FAI), overcoverage of the acetabulum (pincer-type FAI), or both (mixed-type FAI) cause mechanical abutment between the femoral neck and the acetabular rim. Although cam-type FAI is more commonly seen in young males, pincer-type FAI is more likely to become symptomatic in middle-aged women. In cam-type FAI, damage to the acetabular labrum, along with abrasion and/or delamination of the articular cartilage, is typically seen in the anterior-to-superior aspect of the joint, where the femoral head asphericity ("bump") can increase shear loading, most commonly during hip flexion and/or internal rotation $[1,10]$. A so-called contre-coup lesion can be observed occasionally in pincer-type FAI [1]. In this case, a pathologic lever mechanism at the anterior acetabular rim during hip flexion can lead to levering of the femoral head against the posteroinferior acetabular wall, causing cartilage damage in this region.

Symptomatic FAI, if left untreated, may cause early osteoarthritis (OA) [9, 15]. However, it is not yet fully understood what sort of FAI-related damage to the joint (including cartilage damage, labral tears, and synovitis) is most likely to lead to symptoms. Furthermore, it remains unclear why this may lead to symptoms in some patients but not in others, because the prevalence of asymptomatic patients with cam and pincer deformities has been reported as high as $37 \%$ to $68 \%$ [8]. In symptomatic cases, reliable and reproducible assessment of the damage to the articulating surfaces and the labrum allows not only for improvement in the decision-making process of treating physicians concerning joint preservation surgery or joint replacement, but also will facilitate monitoring of surgical outcomes and cartilage status over time.

MRI has become the gold standard for cartilage imaging in the hip. In addition to morphologic sequences, various biochemically sensitive techniques have evolved, each targeting different biochemical changes in OA onset and progression [3, 11, 18, 26, 33].
The loss of cartilage glycosaminoglycan (GAG) is understood as an early and potentially reversible change in the OA course [4]. The well-established technique of delayed gadolinium-enhanced MRI of cartilage (dGEMRIC) has been shown to correlate inversely with the cartilage GAG content [5]. After application of a gadolinium-based contrast agent, the negatively charged gadolinium penetrates into the cartilage. In regions with cartilage degeneration and GAG loss, the gadolinium uptake is higher and vice versa. As gadolinium reduces T1 relaxation $\left(\mathrm{T} 1_{\mathrm{Gd}}\right)$, regions with lower GAG content and presumably higher cartilage degeneration appear with lower $\mathrm{T} 1_{\mathrm{Gd}}$ values than the healthy, GAG-rich counterparts. However, with potential risks associated with the use of gadolinium, including nephrogenic systemic fibrosis [12] and gadolinium deposition [25], noncontrast-based cartilage imaging techniques would offer substantial benefits for future applications.

T2 mapping offers the ability to detect subtle cartilage changes without the need for contrast media application. In previous studies, this imaging technique has been shown to be sensitive to cartilage water content and collagen fiber orientation [18]. With early progressive cartilage degeneration, $\mathrm{T} 2$ may pick up alterations within the cartilage hydration and collagen fiber integrity $[16,20]$. Of note, diurnal effects and loading conditions before imaging have to be considered when T2 mapping is performed, because diurnal variations in the cartilage water content and loading-induced rearrangement of the collagen fiber network might alter $\mathrm{T} 2$ relaxation $[19,21]$. Notably, for hip cartilage, regional differences in T2 relaxation values might be attributed to the magic angle effect, which is an increase in T2 relaxation when tightly bound collagen bundles are orientated at $55^{\circ}$ to the main magnetic field [32]. Especially in the curved cartilage regions of the hip, this effect is difficult to avoid. However, as a result of this phenomenon, regional differences in $\mathrm{T} 2$ relaxation might not be the result of structural changes and must be taken into account when regional and zonal distribution of $\mathrm{T} 2$ relaxation in the hip is interpreted.

The purpose of this study was to answer the following questions: (1) Are dGEMRIC and T2 mapping analyses both capable of detecting cartilage damage in a region of the hip that is prone to damage in FAI? (2) Is there a correlation between these two techniques for acetabular and femoral cartilage assessment?

\section{Patients and Methods}

Institutional review board approval was obtained before conducting this study.

Our study group consisted of 31 patients with symptomatic FAI (32 hips, 16 women, 15 men; mean age, $27 \pm$ 
8 years; range, $16-47$ years, 18 right hips, 14 left hips). An initial database search for all patients who received a 3-T dGEMRIC scan with an additional T2 mapping sequence at our institution between December 2013 and February 2015 (inclusive) yielded 269 patients. We narrowed this cohort by excluding those patients who had a mislabeled scan type or lacked certain necessary sequences $\left(\mathrm{T} 1_{\mathrm{Gd}}\right.$ or $\left.\mathrm{T} 2\right)(\mathrm{n}=$ 24); had a near-normal or normal hip morphology $(n=46)$; had inflammatory, genetic/developmental, necrotic, and/or malignant bone conditions (eg, avascular necrosis, Perthes, multiple epiphyseal dysplasia, rickets, osteochondroma, etc) $(\mathrm{n}=36)$; had isolated hip dysplasia $(\mathrm{n}=23)$; had severe osteoarthritis without measurable joint space $(\mathrm{n}=$ $3)$; had isolated labral tears $(\mathrm{n}=3)$; had strictly postoperative scans $(\mathrm{n}=26)$; and had absent or incomplete surgical chondromalacia grades $(n=76)$. The final cohort of 32 hips comprised the following types of radiographic conditions by fraction: 18 hips (56.3\%) with a cam lesion only, three hips $(9.4 \%)$ with a pincer lesion only, nine hips (28.1\%) with a combined (cam + pincer) lesion, and two hips (6.3\%) with cam FAI and borderline/mild hip dysplasia.

The control group consisted of 10 asymptomatic volunteers (10 hips, seven women, three men; mean age, $28 \pm$ 3 years; range, 24-33 years, five right hips, five left hips). Before their enrollment, each individual of the control group provided written informed consent. The WOMAC was administered to rule out any hip-related pain symptoms. No volunteer underwent prior hip surgery or had any contraindications to undergo hip MRI.

All MR images were acquired in a sagittal imaging plane on a 3-T system (Magnetom Trio; Siemens Medical Solutions, Erlangen, Germany) with a flexible surface coil (four-channel flex coil; Siemens Medical Solutions). Patients (symptomatic cohort) and volunteers (asymptomatic cohort) were examined in the same supine position. For morphologic cartilage assessment, a proton density-weighted fat-suppressed sequence with a slice thickness of $3 \mathrm{~mm}$ was used. All patients underwent the intravenous injection of a double-dose solution of gadolinium-based contrast agent $(0.2 \mathrm{mmol} / \mathrm{kg} \mathrm{Gd}-$ DTPA $^{2-}$, MAGNEVIST ${ }^{\circledR}$; Bayer Healthcare, Whippany, NJ, USA). As a result of ethical reasons around potential needless harm, the volunteers did not receive contrast injections. According to previously published protocols [17], each patient underwent a physical exercise protocol to ensure gadolinium uptake into the cartilage. $\mathrm{T} 1_{\mathrm{Gd}}$ maps were then generated 45 minutes after contrast media administration. A dual-flip angle, three-dimensional gradient-echo acquisition with a B1 field mapping sequence was used. Repetition time (TR) and echo time (TE) for each acquisition were $15 \mathrm{~ms}$ and $8 \mathrm{~ms}$, respectively. Flip angles of $5^{\circ}$ and $25^{\circ}$ were selected. Field of view (FOV) was set to $160 \mathrm{~mm}$ with a matrix size of $384 \mathrm{~mm}$, resulting in a $0.4-\mathrm{mm}$ in-plane resolution. Slice thickness was 3 $\mathrm{mm}$, and 88 slices were acquired to cover the entire cartilage surface. To decrease possible confounding factors as a result of loading conditions, T2 maps were generated at the end of each scan. T2 maps were acquired using a two-dimensional multislice multiecho spin-echo sequence. Five echoes were acquired with TEs of 14, 28, 41,55 , and $67 \mathrm{~ms}$ and a TR of $1140 \mathrm{~ms}$. The FOV was 160 $\mathrm{mm}$ with a matrix size of $320 \mathrm{~mm}$, resulting in $0.5-\mathrm{mm}$ inplane resolution. Slice thickness was $4 \mathrm{~mm}$ and 16 slices were acquired. T2 cartilage mapping was done by voxelwise estimation of the T2 from the last four echoes (TE $=28,41,55,67 \mathrm{~ms}$ ). The first echo was not used because it is affected by stimulated echoes, which occur on top of spin echoes as a result of imperfect slice selection gradients resulting from B1 field inhomogeneities. Before quantification, the T2 maps were postprocessed to increase their reliability using a constrained variable projection optimization method.

Image analysis was carried out using Synapse 3D software (Fujifilm Corp, Tokyo, Japan). Morphologic cartilage assessment was carried out in the sagittal and coronal planes by an orthopaedic surgeon (Y-JK) with several years of experience in morphologic and biochemical cartilage imaging. Full-thickness acetabular and femoral head cartilages were graded for each hip according to a modified Outerbridge [24] classification system $(0=$ normal cartilage; 1 = partial cartilage degeneration; 2 = full-thickness cartilage degeneration). In both planes, the chondrolabral transitional zone was identified (Fig. 1). In a midsagittal plane, $\mathrm{T} 1, \mathrm{~T} 1_{\mathrm{Gd}}$, and $\mathrm{T} 2$ maps were referenced and regionof-interest (ROI) analysis was carried out in acetabular and corresponding femoral head cartilage from the chondrolabral transitional zone reaching approximately 3 to $6 \mathrm{~mm}$ toward the acetabular fossa/fovea capitis femoris in each hip (Fig. 2). The corresponding region on the femoral head was meant to serve as an internal control so as to standardize the acetabular signals among symptomatic hips in patients and asymptomatic hips in volunteer control subjects. In cases of advanced cartilage degeneration in this region, cartilage analysis was conducted in the same plane, but more centrally in an area that allowed for reliable ROI placement within cartilage boundaries. All relaxation measures are provided as mean $\pm \mathrm{SD}$.

Morphologic assessment of acetabular cartilage in the symptomatic group yielded 10 hips that were graded as Outerbridge 0 and 22 hips that were graded as Outerbridge 1. Meanwhile, femoral head cartilage was graded as Outerbridge 0 in 29 hips and Outerbridge 1 in three hips. In the asymptomatic group, morphologic cartilage assessment revealed Outerbridge 1 changes in two hips for acetabular cartilage and Outerbridge 0 for all femoral head cartilages. 


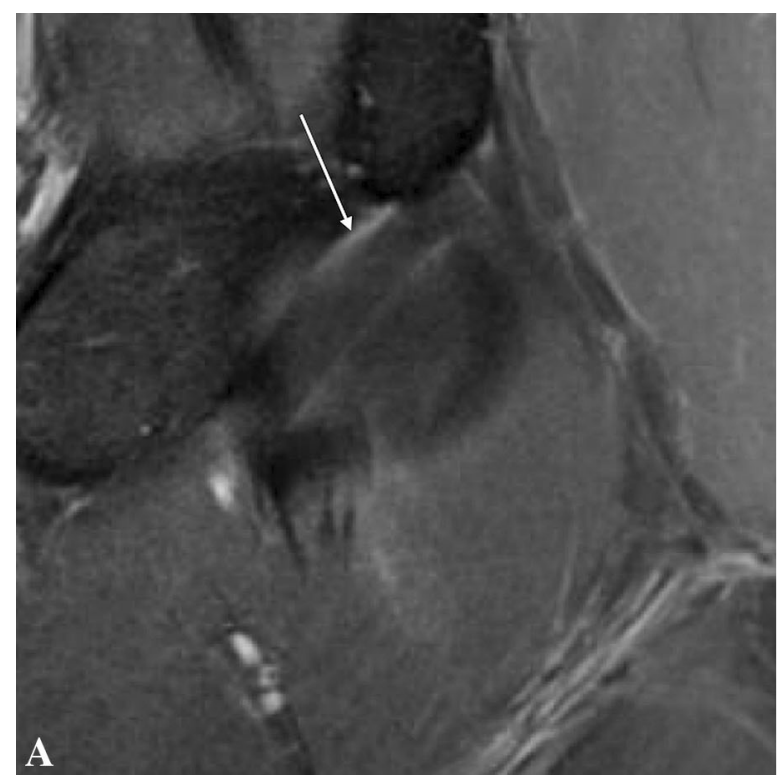

Fig. 1A-B MRI of a 36-year-old female patient with FAI is shown. In the coronal plane $(\mathbf{A})$ and sagittal plane $(\mathbf{B})$, the chondrolabral transitional zone was identified. In this case, a labral tear (arrows) was

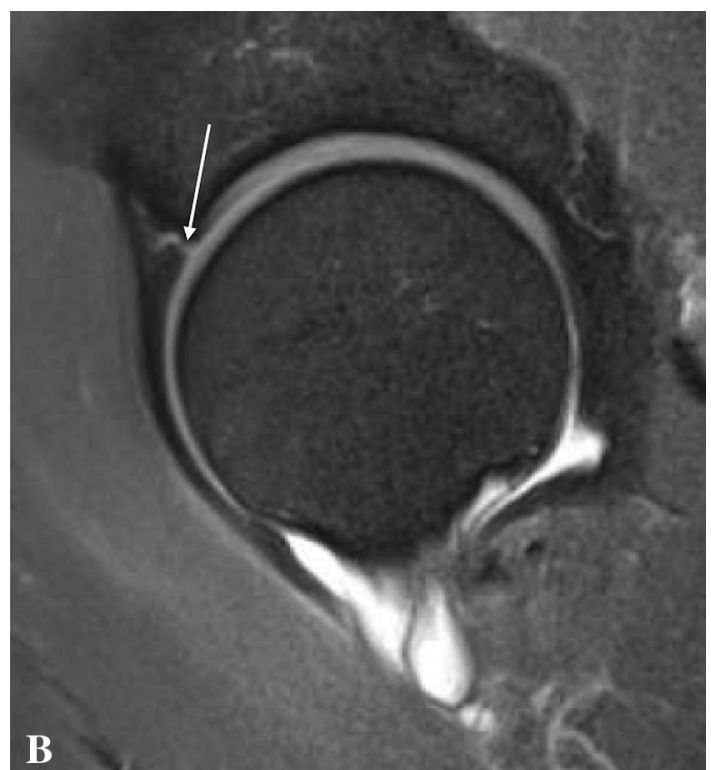

noted that allowed for clear differentiation between the labrum and acetabular cartilage.

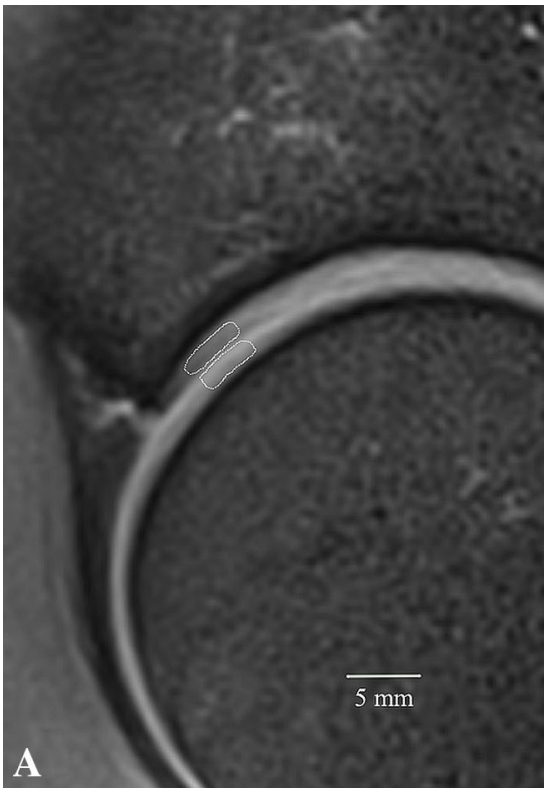

Fig. 2A-C In the midsagittal plane, the proton density-weighted fatsuppressed sequence for morphologic cartilage assessment $(\mathbf{A}), \mathrm{T}_{\mathrm{Gd}}$ (B), and T2 maps (C) was referenced. Region of interest analysis was conducted in acetabular and corresponding femoral head cartilage from the chondrolabral transitional zone reaching approximately 3 to

\section{Statistical Analysis}

For comparison of acetabular and femoral $\mathrm{T} 1, \mathrm{~T} 1_{\mathrm{Gd}}, \mathrm{T} 2$, and modified Outerbridge values, a Student's t-test was used. Pearson's correlation coefficient (r) along with 95\%
$6 \mathrm{~mm}$ medially. Notably, both color-scaled maps picked up acetabular cartilage damage in this region that was not detected during morphologic cartilage assessment.

confidence interval (CI) was calculated for a correlation between acetabular and femoral head measures of $\mathrm{T} 1$, $\mathrm{T} 1_{\mathrm{Gd}}$, and $\mathrm{T} 2$ values. A $\mathrm{p}$ value $<0.05$ was considered to be statistically significant. 


\section{Results}

Both dGEMRIC and T2 mapping analyses at 3-T resolution were capable of detecting cartilage damage at the chondrolabral junction in the anterior-peripheral sector of the acetabulum, a region that is prone to damage in FAI. In the symptomatic cohort, dGEMRIC analysis revealed lower $\mathrm{T} 1_{\mathrm{Gd}}$ values in acetabular cartilage when compared with corresponding femoral head cartilage $(515 \pm 165 \mathrm{~ms}$ versus $650 \pm 191 \mathrm{~ms}, \mathrm{p}<0.001)$. Similarly for T2 mapping analysis, lower T2 relaxation values were found in acetabular cartilage when compared with corresponding femoral head cartilage $(39 \pm 8 \mathrm{~ms}$ versus $46 \pm 10 \mathrm{~ms}, \mathrm{p}<0.001)$. Meanwhile in the asymptomatic cohort, analysis of acetabular and femoral head cartilage yielded no differences with the numbers available in terms of $\mathrm{T} 1(861 \pm 130 \mathrm{~ms}$ versus $860 \pm$ $182 \mathrm{~ms}, \mathrm{p}=0.98)$ and $\mathrm{T} 2$ values $(43 \pm 7 \mathrm{~ms}$ versus $42 \pm$ $6 \mathrm{~ms}, \mathrm{p}=0.73$ ) (Fig. 3). When comparing T2 values in symptomatic and asymptomatic cohorts, no difference was observed with the numbers available for either acetabular cartilage $(39 \pm 8 \mathrm{~ms}$ versus $43 \pm 7 \mathrm{~ms}, \mathrm{p}=$ $0.15)$ or femoral head cartilage $(46 \pm 10 \mathrm{~ms}$ versus $42 \pm$ $6 \mathrm{~ms}, \mathrm{p}=0.25)$.
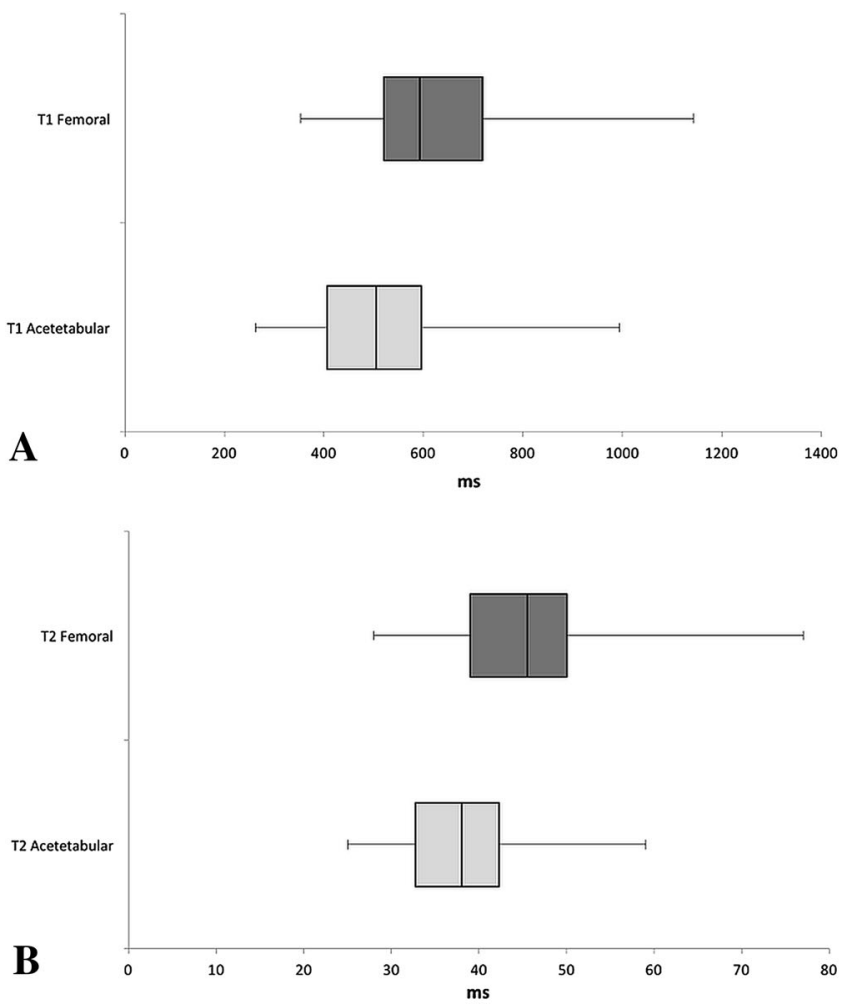

Fig. 3A-D Box plot showing the median, first and third quartiles, and the range of $\mathrm{T} 1_{\mathrm{Gd}}$ values $(\mathbf{A})$ and $\mathrm{T} 2$ mapping values $(\mathbf{B})$ in patients with FAI. Notably, lower values were observed for acetabular
Despite the observed ability of both 3-T dGEMRIC and $\mathrm{T} 2$ in specifically distinguishing damaged from relatively undamaged hip cartilage in the symptomatic cohort, these two techniques did not appear to correlate with each other. For the symptomatic cohort, no correlation with the numbers available was noted between $\mathrm{T} 1_{\mathrm{Gd}}$ and $\mathrm{T} 2$ values for acetabular cartilage $(\mathrm{r}=-0.22 ; 95 \% \mathrm{CI},-0.75$ to $0.48 ; \mathrm{p}=$ $0.54)$ as well as for femoral head cartilage $(r=-0.40 ; 95 \%$ $\mathrm{CI},-0.82$ to $0.31 ; \mathrm{p}=0.26$ ). A similar lack of correlation was observed in the asymptomatic cohort for acetabular cartilage ( $\mathrm{r}=-0.05 ; 95 \% \mathrm{CI},-0.39$ to $0.30 ; \mathrm{p}=0.79)$ and femoral head cartilage $(\mathrm{r}=-0.24 ; 95 \% \mathrm{CI},-0.54$ to 0.12 ; $\mathrm{p}=0.18$ ). Both $\mathrm{T} 1_{\mathrm{Gd}}$ and $\mathrm{T} 2$ did not differ with the numbers available between Outerbridge 1 hips and Outerbridge 0 hips for acetabular cartilage $\left(\mathrm{T}_{\mathrm{Gd}}\right.$ : $482 \pm 146 \mathrm{~ms}$ versus $588 \pm 186 \mathrm{~ms}, \mathrm{p}=0.09 ; \mathrm{T} 2: 39 \pm 9 \mathrm{~ms}$ versus $38 \pm 4 \mathrm{~ms}, \mathrm{p}$ $=0.76)$.

\section{Discussion}

dGEMRIC has been shown to be a reliable and reproducible tool for biochemical cartilage assessment $[2,14,33]$. However, with physiological differences in the
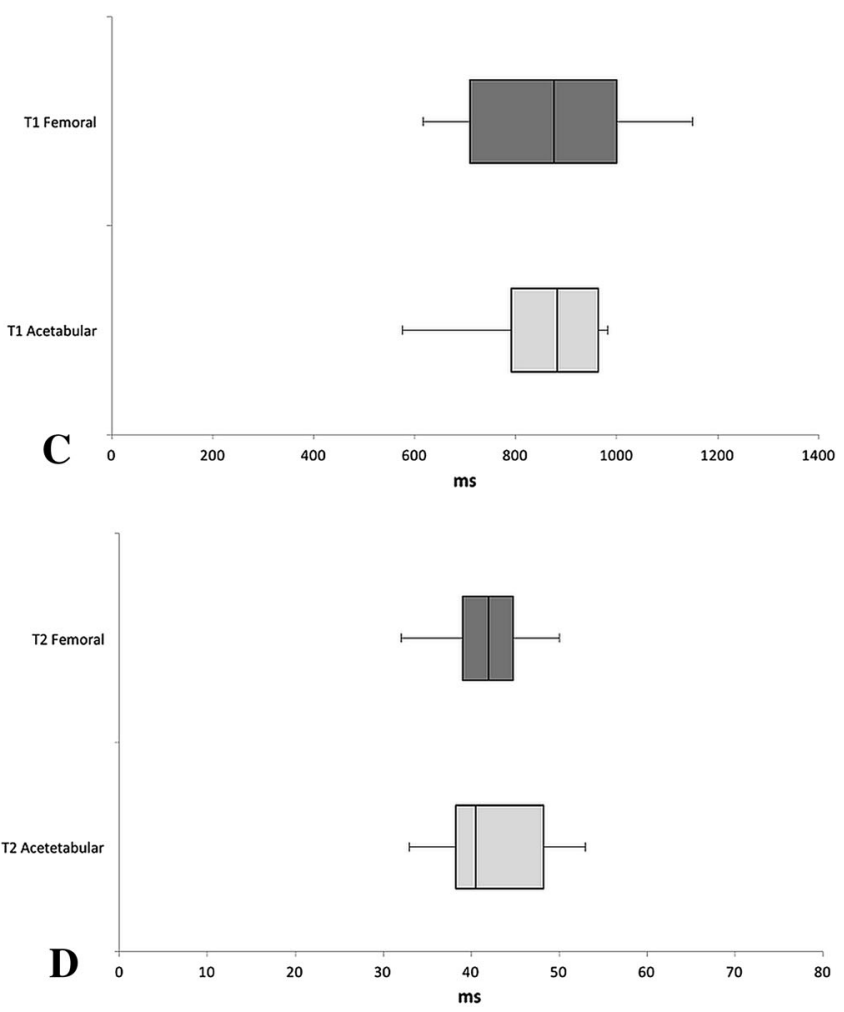

cartilage when compared with femoral head cartilage. In the control cohort, similar values for T1 values $(\mathbf{C})$ and T2 mapping values (D) were noted. 
GAG distribution among weightbearing and nonweightbearing regions, $\mathrm{T} 1_{\mathrm{Gd}}$ values have been noted to vary among different regions of the hip [34]. As for T2 mapping, physical MR characteristics such as the magic angle effect and differences in the microstructure and composition of articular cartilage can lead to physiological regional differences and zonal stratification of T2 values. Therefore, a reliable interpretation of $\mathrm{T} 2$ can be difficult when comparing between patients and different joint regions, and results should be scrutinized thoroughly to prevent misinterpretation and possible false treatment decisions. In early stages of FAI, a regional cartilage injury pattern is assumed with cartilage damage predominantly in the anterior aspect of the acetabulum, where the mechanical abutment between the femoral neck and the acetabular rim can cause direct damage to the cartilage $[1,10]$. With this study, we aimed to directly compare the two imaging techniques of dGEMRIC and T2 mapping for analysis of acetabular and corresponding femoral head cartilage in this distinct cartilage region of the hip. In the symptomatic cohort, both the dGEMRIC index and T2 mapping values were found to decrease in acetabular cartilage when compared with corresponding femoral head cartilage. This observation was not seen in the control group, in which no differences in $\mathrm{T} 1$ or T2 were noted between the two corresponding cartilage regions with the numbers available. To clarify, the T1 measurements in the control group served a quality control purpose to rule out any acquisition-related issues. Having highly comparable acetabular and femoral head T1 values validates our general scan methodology. We did not observe a correlation between the two imaging methodologies, indicating that the biochemical changes that occur during cartilage degeneration, including GAG content depletion, collagen fiber anisotropy, and cartilage water content depletion, are complex.

We acknowledge the limitations of this study. First, we did not analyze intra- and interobserver agreement. However, cartilage assessment was done by a single observer with several years of experience in morphologic and biochemical cartilage imaging. Given the number of patients in our study, we believe that it is unlikely that a lack of a second reader biased our results. Moreover, only a limited number of ROIs was analyzed. However, this study does not aim to provide a subregional analysis of $\mathrm{T} 1, \mathrm{~T} 1_{\mathrm{Gd}}$, and T2 data for the entire hip. The goal of this study was to accurately assess and compare these two imaging techniques in a specific region of acetabular and femoral cartilage that is prone to damage in FAI. Although this study was conducted on a 3-T MR scanner that allowed for differentiation between the two cartilage layers, and close attention was paid to ensure ROI placement within cartilage boundaries, we cannot rule out the possibility that inclusion of joint fluid and effusion, especially with cleft formation in cartilage degeneration, might have influenced the $\mathrm{T} 1_{\mathrm{Gd}}$ and $\mathrm{T} 2$ analysis. Additionally, a possible influence of gadolinium administration on $\mathrm{T} 2$ relaxation (FAI cohort) might have biased our results. However, in analyzing the effect of intraarticular gadolinium administration on $\mathrm{T} 2 *$ relaxation, a previously published study has not detected such an influence [23]. The fact that we did not observe a significant difference when comparing T2 values across healthy and unhealthy hips highlights the complexity of interpreting this methodology. As such, a simple comparison of $\mathrm{T} 2$ values might not be useful and a more nuanced approach, including an internal control, might be helpful to better understand $\mathrm{T} 2$ measurements.

As a result of the absence of baseline (precontrast) T1 assessment in current dGEMRIC protocols, few studies provide data on precontrast T1 values. Although previously reported precontrast $\mathrm{T} 1$ values for knee cartilage of 20 asymptomatic volunteers [30] were reported to be higher than our results (ranging from $1146 \pm 133 \mathrm{~ms}$ to $1184 \pm$ $100 \mathrm{~ms}$ ), our findings are in keeping with those of others. In a group of 10 asymptomatic volunteers, Bittersohl et al. [2] reported mean precontrast $\mathrm{T} 1$ values of $846 \pm 96 \mathrm{~ms}$ for hip cartilage. Notably, these values were obtained from acetabular and femoral cartilage of the weightbearing zone in four coronal reformats at $1.5 \mathrm{~T}$. In a higher field strength scanner, the $\mathrm{T} 1$ values are expected to be higher. For dGEMRIC analysis in FAI cohorts, Domayer et al. [6] reported mean dGEMRIC values of $546 \mathrm{~ms}$ in a cohort of 20 patients with FAI. Although this study was also conducted on a 1.5-T MR scanner, which did not allow for reliable separation of acetabular and femoral cartilages, the typical cartilage injury pattern observed in FAI was somewhat reflected by their results, because lower values were observed in peripheral acetabular cartilage when compared with central cartilage. Furthermore, with advanced degeneration (dGEMRIC index $<500 \mathrm{~ms}$ ), a more localized decrease of $\mathrm{T} 1_{\mathrm{Gd}}$ in the anterosuperior sector was noted. In a dGEMRIC-based study of hips with FAI by Zilkens et al. [34], seven radial reformats of each hip were created. Their results further supported regional differences in GAG distribution within the hip because higher dGEMRIC values were noted in weightbearing cartilage. Of note, this regional distribution pattern was only observed in acetabular cartilage, whereas the femoral head cartilage revealed only minor variation with location. This information is critical and has to be considered when $\mathrm{T} 1$ analysis within the acetabular hemisphere is conducted.

As a result of physiological differences in cartilage composition among weightbearing and nonweightbearing cartilage, diurnal effects, regional load distribution during motion, and dependence of $\mathrm{T} 2$ values with orientation to the magnetic field, regional T2 mapping analysis for hip cartilage might be even more critical. However, Subburaj 
et al. [28] postulated that regional T2 analysis in the hip is more sensitive than global T2 measures for discrimination between subjects with and without FAI. In that study (nine patients with FAI, 12 control subjects), T2 values in the anterosuperior region were noted to be higher than global ROIs $(26 \pm 3 \mathrm{~ms}$ versus $31 \pm 2 \mathrm{~ms} ; \mathrm{p}<0.001)$. Given these phenomena, previously reported $\mathrm{T} 2$ mapping values in presumably healthy hip cartilage of control groups provide evidence for a natural variation of $\mathrm{T} 2$ relaxation in different regions of the joint as well as between femoral and acetabular cartilage. Ho et al. [13] analyzed the regional T2 distribution in hips of asymptomatic volunteers. Although mean T2 values for acetabular $(45-53 \mathrm{~ms})$ and femoral cartilage (47-54 ms) are comparable (and similar to our control group), important differences between the investigated subregions were noted. Although the highest $\mathrm{T} 2$ values were noted in the superoposterior region for femoral cartilage, T2 values in acetabular cartilage were the highest in anteroinferior cartilage. For superoanterior cartilage (somewhat comparable to the ROI in this study), slightly higher values were observed for femoral cartilage ( $51 \pm 5 \mathrm{~ms}$ versus $46 \pm 5 \mathrm{~ms}$ ).

Contrary to these results, Watanabe et al. [29] noted a topographic variation in $\mathrm{T} 2$ values with increasing $\mathrm{T} 2$ relaxation toward the magic angle. Notably, this was observed for femoral cartilage and ROI analysis was only conducted in the upper hemisphere of the hip. The influence of ongoing cartilage degeneration on $\mathrm{T} 2$ relaxation is yet not completely understood. Nishii et al. [22] reported that the physiological zonal stratification pattern of $\mathrm{T} 2$ relaxation is less apparent with cartilage degeneration. However, although previously reported studies noted an increase of $\mathrm{T} 2$ relaxation with cartilage damage [28, 31], others [7] (including our results) noted lower T2 values in regions with presumable cartilage damage. Siebenrock et al. [27] recently published their results on $\mathrm{T} 2$ and $\mathrm{T} 2 *$ relaxation in an ovine FAI model. In that study, T2 and T2* analyses of six different regions of the acetabulum were compared with histologic grades (Mankin score) of cartilage degeneration. Interestingly, a negative correlation between histologic cartilage grading and $\mathrm{T} 2$ analysis $(\mathrm{r}=$ $-0.79, \mathrm{p}<0.001)$ was found. Although this study was conducted in an ovine model and transferring these results to humans might be critical, interpreting these to some extent diverging results seems difficult. Possibly, at a certain stage of the injury, direct damage to acetabular cartilage as is seen in FAI might lead to a decrease in T2 relaxation, whereas other cartilage disease patterns might lead to increasing $\mathrm{T} 2$ in early stages.

In conclusion, the two biochemical-sensitive imaging modalities, dGEMRIC and T2 mapping, show different results at $3 \mathrm{~T}$ for hip cartilage assessment in regions that are prone to damage in FAI. Therefore, we believe that both methodologies may serve as potential biomarkers for cartilage assessment in patients with FAI. With T2 in particular, although the direct values do not appear to contain the damage signal that would distinguish unhealthy and healthy hip cartilage, its internal comparison to healthy cartilage within the same hip appears to do so. Published results on topographic $\mathrm{T} 2$ variation need to be considered to not erroneously misjudge the cartilage status and the use of internal healthy control cartilage could be beneficial. Future studies, including larger study cohorts and other nongadolinium-based methodologies in this specific patient population, are mandatory to further reaffirm our findings.

Acknowledgments We thank the Department of Orthopaedic Surgery at Boston Children's Hospital for providing funding for the volunteer MR imaging.

\section{References}

1. Beck M, Kalhor M, Leunig M, Ganz R. Hip morphology influences the pattern of damage to the acetabular cartilage: femoroacetabular impingement as a cause of early osteoarthritis of the hip. J Bone Joint Surg Br. 2005;87:1012-1018.

2. Bittersohl B, Hosalkar HS, Kim YJ, Werlen S, Siebenrock KA, Mamisch TC. Delayed gadolinium-enhanced magnetic resonance imaging (dGEMRIC) of hip joint cartilage in femoroacetabular impingement (FAI): are pre- and postcontrast imaging both necessary? Magn Reson Med. 2009;62:1362-1367.

3. Borthakur A, Mellon E, Niyogi S, Witschey W, Kneeland JB, Reddy R. Sodium and T1rho MRI for molecular and diagnostic imaging of articular cartilage. NMR Biomed. 2006;19:781-821.

4. Burstein D, Bashir A, Gray ML. MRI techniques in early stages of cartilage disease. Invest Radiol. 2000;35:622-638.

5. Burstein D, Gray M, Mosher T, Dardzinski B. Measures of molecular composition and structure in osteoarthritis. Radiol Clin North Am. 2009;47:675-686.

6. Domayer SE, Mamisch TC, Kress I, Chan J, Kim YJ. Radial dGEMRIC in developmental dysplasia of the hip and in femoroacetabular impingement: preliminary results. Osteoarthritis Cartilage. 2010;18:1421-1428.

7. Ferro FP, Ho CP, Dornan GJ, Surowiec RK, Philippon MJ. Comparison of $\mathrm{T} 2$ values in the lateral and medial portions of the weight-bearing cartilage of the hip for patients with symptomatic femoroacetabular impingement and asymptomatic volunteers. Arthroscopy. 2015;31:1497-1506.

8. Frank JM, Harris JD, Erickson BJ, Slikker W 3rd, Bush-Joseph CA, Salata MJ, Nho SJ. Prevalence of femoroacetabular impingement imaging findings in asymptomatic volunteers: a systematic review. Arthroscopy. 2015;31:1199-1204.

9. Ganz R, Leunig M, Leunig-Ganz K, Harris WH. The etiology of osteoarthritis of the hip: an integrated mechanical concept. Clin Orthop Relat Res. 2008;466:264-272.

10. Ganz R, Parvizi J, Beck M, Leunig M, Notzli H, Siebenrock KA. Femoroacetabular impingement: a cause for osteoarthritis of the hip. Clin Orthop Relat Res. 2003;417:112-120.

11. Hesper T, Hosalkar HS, Bittersohl D, Welsch GH, Krauspe R, Zilkens C, Bittersohl B. T2* mapping for articular cartilage assessment: principles, current applications, and future prospects. Skeletal Radiol. 2014;43:1429-1445.

12. Heverhagen JT, Krombach GA, Gizewski E. Application of extracellular gadolinium-based MRI contrast agents and the risk of nephrogenic systemic fibrosis. Rofo. 2014;186:661-669. 
13. Ho CP, Surowiec RK, Ferro FP, Lucas EP, Saroki AJ, Dornan GJ, Fitzcharles EK, Anz AW, Smith WS, Wilson KJ, Philippon MJ. Subregional anatomical distribution of T2 values of articular cartilage in asymptomatic hips. Cartilage. 2014;5:154-164.

14. Lattanzi R, Petchprapa C, Ascani D, Babb JS, Chu D, Davidovitch RI, Youm T, Meislin RJ, Recht MP. Detection of cartilage damage in femoroacetabular impingement with standardized dGEMRIC at 3 T. Osteoarthritis Cartilage. 2014;22:447-456.

15. Leunig M, Ganz R. [Femoroacetabular impingement. A common cause of hip complaints leading to arthrosis] [in German]. Unfallchirurg. 2005;108:9-10, 12-17.

16. Liess C, Lusse S, Karger N, Heller M, Gluer CC. Detection of changes in cartilage water content using MRI T2-mapping in vivo. Osteoarthritis Cartilage. 2002;10:907-913.

17. Mamisch TC, Dudda M, Hughes T, Burstein D, Kim YJ. Comparison of delayed gadolinium enhanced MRI of cartilage (dGEMRIC) using inversion recovery and fast T1 mapping sequences. Magn Reson Med. 2008;60:768-773.

18. Mosher TJ, Dardzinski BJ. Cartilage MRI T2 relaxation time mapping: overview and applications. Semin Musculoskelet Radiol. 2004;8:355-368.

19. Mosher TJ, Smith HE, Collins C, Liu Y, Hancy J, Dardzinski BJ, Smith MB. Change in knee cartilage T2 at MR imaging after running: a feasibility study. Radiology. 2005;234:245-249.

20. Nieminen MT, Rieppo J, Toyras J, Hakumaki JM, Silvennoinen J, Hyttinen MM, Helminen HJ, Jurvelin JS. T2 relaxation reveals spatial collagen architecture in articular cartilage: a comparative quantitative MRI and polarized light microscopic study. Magn Reson Med. 2001;46:487-493.

21. Nishii T, Kuroda K, Matsuoka Y, Sahara T, Yoshikawa H. Change in knee cartilage $\mathrm{T} 2$ in response to mechanical loading. $J$ Magn Reson Imaging. 2008;28:175-180.

22. Nishii T, Shiomi T, Tanaka H, Yamazaki Y, Murase K, Sugano $\mathrm{N}$. Loaded cartilage T2 mapping in patients with hip dysplasia. Radiology. 2010;256:955-965.

23. Nissi MJ, Mortazavi S, Hughes J, Morgan P, Ellermann J. T2* relaxation time of acetabular and femoral cartilage with and without intraarticular gadopentetate dimeglumine in patients with femoroacetabular impingement. AJR Am J Roentgenol. 2015;204:W695-700.

24. Outerbridge RE. The etiology of chondromalacia patellae. 1961. Clin Orthop Relat Res. 2001;389:5-8.
25. Robert P, Violas X, Grand S, Lehericy S, Idee JM, Ballet S, Corot C. Linear gadolinium-based contrast agents are associated with brain gadolinium retention in healthy rats. Invest Radiol. 2016;51:73-82.

26. Schleich C, Bittersohl B, Miese F, Schmitt B, Muller-Lutz A, Sondern M, Antoch G, Krauspe R, Zilkens C. Glycosaminoglycan chemical exchange saturation transfer at $3 \mathrm{~T}$ MRI in asymptomatic knee joints. Acta Radiol. 2016;57:627-632.

27. Siebenrock KA, Kienle KP, Steppacher SD, Tannast M, Mamisch TC, von Rechenberg B. Biochemical MRI predicts hip osteoarthritis in an experimental ovine femoroacetabular impingement model. Clin Orthop Relat Res. 2015;473:13181324.

28. Subburaj K, Valentinitsch A, Dillon AB, Joseph GB, Li X, Link TM, Vail TP, Majumdar S. Regional variations in MR relaxation of hip joint cartilage in subjects with and without femoralacetabular impingement. Magn Reson Imaging. 2013;31:11291136.

29. Watanabe A, Boesch C, Siebenrock K, Obata T, Anderson SE. T2 mapping of hip articular cartilage in healthy volunteers at 3T: a study of topographic variation. $J$ Magn Reson Imaging. 2007;26:165-171.

30. Williams A, Mikulis B, Krishnan N, Gray M, McKenzie C, Burstein D. Suitability of T(1Gd) as the dGEMRIC index at $1.5 \mathrm{~T}$ and 3.0T. Magn Reson Med. 2007;58:830-834.

31. Wyatt C, Kumar D, Subburaj K, Lee S, Nardo L, Narayanan D, Lansdown D, Vail T, Link TM, Souza RB, Majumdar S. Cartilage T1rho and T2 relaxation times in patients with mild-tomoderate radiographic hip osteoarthritis. Arthritis Rheumatol. 2015;67:1548-1556

32. Xia Y. Magic-angle effect in magnetic resonance imaging of articular cartilage: a review. Invest Radiol. 2000;35:602-621.

33. Zilkens C, Jager M, Bittersohl B, Dudda M, Millis MB, Kim YJ, Muhr G, Krauspe R, Mamisch TC. [Delayed gadolinium enhanced MRI of cartilage (dGEMRIC): molecular MRI of hip joint cartilage] [in German]. Orthopade. 2009;38:591599.

34. Zilkens C, Miese F, Kim YJ, Hosalkar H, Antoch G, Krauspe R, Bittersohl B. Three-dimensional delayed gadolinium-enhanced magnetic resonance imaging of hip joint cartilage at 3T: a prospective controlled study. Eur J Radiol. 2012;81: $3420-3425$. 